
rural

\title{
Le rabais britannique et la révision de la PAC. Vers la fin de la solidarité financière?
}

The British rebate and CAP reform: towards the end of financial solidarity

Louis-Pascal Mahé, Hugo Naudet et Marie-Alix Roussillon-Montfort

\section{(2) OpenEdition}

Journals

Édition électronique

URL : http://journals.openedition.org/economierurale/2204

DOI : 10.4000/economierurale.2204

ISSN : 2105-2581

Éditeur

Société Française d'Économie Rurale (SFER)

Édition imprimée

Date de publication : 10 septembre 2007

Pagination : 59-64

ISSN : 0013-0559

Référence électronique

Louis-Pascal Mahé, Hugo Naudet et Marie-Alix Roussillon-Montfort, « Le rabais britannique et la

révision de la PAC. Vers la fin de la solidarité financière? », Économie rurale [En ligne], 300 | Juillet-août 2007, mis en ligne le 12 novembre 2009, consulté le 01 mai 2019. URL : http://

journals.openedition.org/economierurale/2204 ; DOI : 10.4000/economierurale.2204

(c) Tous droits réservés 


\title{
Le rabais britannique et la révision de la PAC Vers la fin de la solidarité financière?
}

\author{
Louis-Pascal MAHÉ • Professeur E., Agrocampus (École nationale supérieure agronomique) de Rennes \\ Hugo NAUDET • Ingénieur agro-économiste, \\ Agrocampus (École nationale supérieure agronomique) de Rennes \\ Marie-Alix ROUSSILLON-MONTFORT • Ingénieur agro-économiste, ministère de l'Économie, \\ de I'Industrie et de l'Emploi'
}

La remise en cause du rabais britannique fut refusée lors de discussions budgétaires 2007-2013. Sans doute convient-il de voir dans cet épisode classique l'amorce d'une nouvelle réflexion sur la PAC, en particulier sur son coût et son principe de solidarité financière. Dans l'agenda de Lisbonne, les États membres soulignaient le décalage entre les dépenses allouées à l'agriculture et celles en faveur de la compétitivité de l'Union européenne. Louis-Pascal Mahé, Hugo Naudet et Marie-Alix Roussillon-Montfort analysent l'exception britannique, son impact dans les transferts des fonds européens et son rôle dans une éventuelle révision de la PAC. L'objectif de la cohésion européenne serait-il alors maintenu ?

L ors du sommet européen de juin 2005 consacré aux perspectives budgétaires 2007-2013, le Premier ministre britannique Tony Blair refusa la remise en cause du rabais financier accordé au Royaume-Uni (RU), si le financement de la PAC n'était pas réorganisé avant 2013. La tension habituelle entre la France et le RU s'éleva de quelques degrés, particulièrement entre Tony Blair et Jacques Chirac. La discussion financière ne put aboutir et fut transmise à la présidence suivante. N'était-ce là qu'un épisode de plus de l'opposition franco-britannique en matière de politique agricole, un classique européen, ou l'amorce d'une réelle remise en cause de la PAC elle-même, de son coût, et du principe de solidarité financière?

Lors du conseil de décembre 2005, le compromis final de la présidence britannique portait une clause de révision invitant la Commission «à entreprendre un réexamen complet et global, couvrant tous les aspects des dépenses de l'UE, y compris la politique agricole commune, ainsi que des ressources, y compris la compensation en faveur du Royaume-Uni et à faire un rapport en 2008-2009». Cette procédure concer- nant la PAC a été validée en 2006 par l'ensemble des instances européennes sous le nom de « Bilan de santé ». La Commission a publié fin novembre 2007 une brève note proposant des aménagements assez mineurs de la réforme de 2003, mais une large consultation est en cours ${ }^{2}$. La PAC est de nouveau remise en question par plusieurs États membres, d'autant que la stratégie de Lisbonne a souligné le décalage entre les dépenses allouées à l'agriculture et celles en faveur de la compétitivité de l'Union.

Dans ce contexte, deux questions se posent. 1. Le rabais britannique est-il toujours justifié au point de valider le lien entre la révision du rabais et celle de la PAC ?

2. La solidarité financière dans la PAC, source permanente de blocages politiques, n'est-elle pas condamnée ?

1. Les propos tenus dans cet article n'engagent que leurs auteurs et non les institutions auxquelles ils appartiennent.

2. André R. et Laffineur M. (2005). Rapport d'information $n^{\circ} 2367$ sur les perspectives financières 2007-2013, Délégation de l'Assemblée nationale pour l'UE, 9 juin. 


\section{Le rabais, le coût de la PAC pour le Royaume-Uni et l'exception britannique}

Le principe d'un mécanisme de compensation du RU remonte au temps de son adhésion et au Conseil de Dublin en 1975. Le conseil de Fontainebleau de 1984 précisa que les deux tiers de sa contribution nette aux « dépenses affectées » seraient remboursés. Ce remboursement était financé par les autres États membres selon leur clé budgétaire, celle de l'Allemagne étant réduite d'un tiers. Le mécanisme fut modifié par la suite, plusieurs fois mais toujours maintenu. En 1999, au sommet de Berlin les contributions de l'Autriche, de l'Allemagne, de la Suède et des Pays-Bas furent encore réduites ${ }^{3}$. Le caractère très particulier du régime accordé au RU dans cette compensation des balances nettes est patent car il ne modifie qu'assez peu les gains ou pertes des divers États membres mais beaucoup celui du RU. La Commission $^{4}$ a ainsi calculé que sur la période 2008-2013 le solde budgétaire négatif moyen du RU passerait de 0,62 \% du PNB à $0,25 \%$ selon les règles du rabais en vigueur, soit une « compensation » de 0,37 point de PNB. Pour les pays partiellement exempts du financement de la compensation cités ci-dessus, l'effet de la compensation sur leur solde net est inférieur à 0,03 point de PNB. Pour les autres contributeurs nets (Italie, France, Danemark, Chypre, Finlande) la compensation leur coûte environ 0,10 point de PNB.

Ce traitement particulier est-il défendable en raison d'une situation particuliè-

3. À un quart de leur niveau normal: European Commission (1998) - Financing the European Union, Commission report on the operation of the own resources system Directory General XIX, Brussels, 07/10/1998.

4. European Commission (2004). Financing the European Union. Commission report on the operation of the own resources system, COM (2004) 505 final, volumes I and II. rement défavorable du RU résultant des transferts financiers dus à l'ensemble des politiques communes? L'un des principes admis, au moins depuis le conseil de Fontainebleau, est que tout pays supportant un poids budgétaire excessif au regard de sa prospérité relative peut bénéficier d'une correction. Le principe de cohésion valide également des transferts entre régions ou pays dans la mesure où ils réduisent les inégalités et favorisent la convergence. Quels seraient les effets redistributifs du système des dépenses communautaires dans l'Europe des 25 au cours de la période 2007-2013, en l'absence de mécanisme de correction et de rabais britannique ? La corrélation négative entre les soldes nets relatifs avant correction (en \% du PNB) et le niveau de prospérité (revenu par tête) est clairement illustrée par le graphique 1. La dispersion des pays autour de la droite de régression est assez remarquablement faible. Chez les contributeurs nets, les cas particuliers sont Chypre et, dans une mesure moindre, l'Allemagne (plus pénalisés que la moyenne) et le Danemark (plus favorisé). Chez les bénéficiaires d'un solde net positif, l'Irlande, la Grèce, la République Tchèque et la Lituanie sont avantagés, mais Malte est moins bien traitée que son niveau de revenu le prévoirait ${ }^{5}$. Le Royaume-Uni étant au-dessus de la droite, sa contribution nette pourrait encore s'alourdir sans pouvoir être considérée comme pénalisante selon ce principe de redistribution. Au regard du mécanisme financier global de l'UE, il apparaît donc que ce n'est pas le RU qui est particulièrement défavorisé mais au contraire l'Allemagne, Chypre ou Malte.

À l'origine, après de difficiles négociations lors d'adhésion du RU, la compensation fut justifiée par les conséquences

5. On notera l'absence de la Belgique et du Luxembourg, grands bénéficiaires apparents du fait de la présence des Institutions européennes, et qui seraient hors du graphique. 


\section{Graphique 1. Les effets redistributifs du système financier communautaire} (avant rabais britannique, 2007-2013)

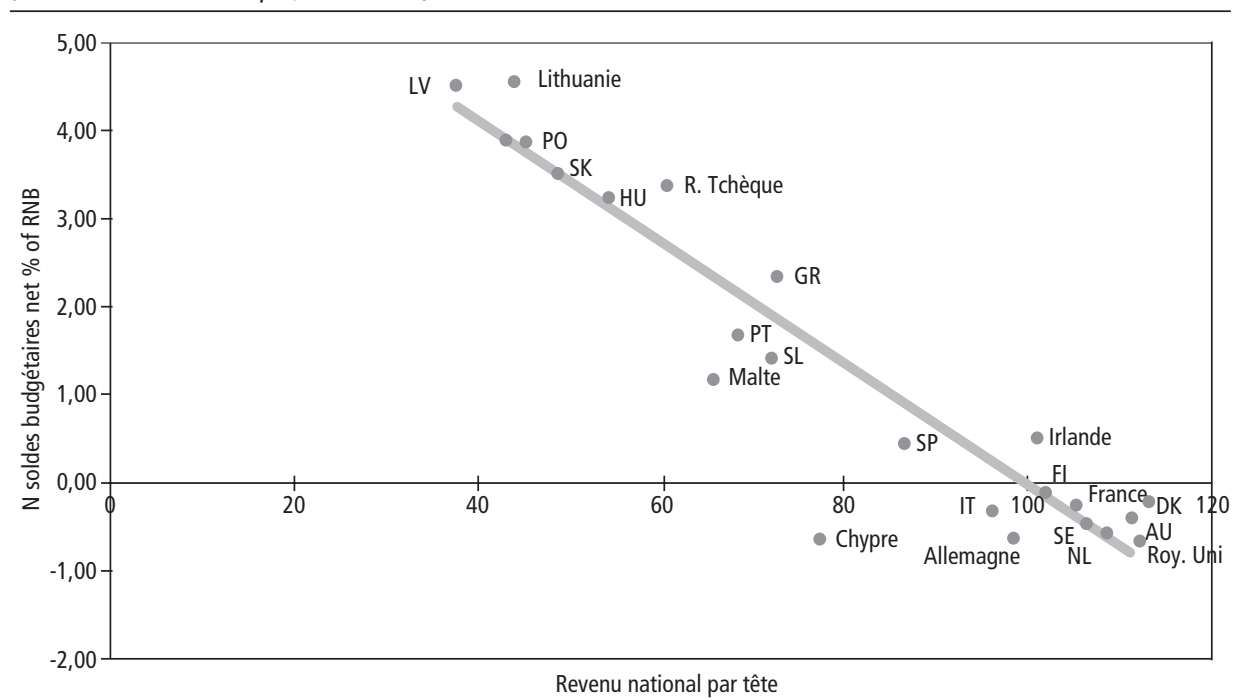

Source : données tirées de COM (2004) 505 Annexe table II.

négatives réelles de la PAC pour ce pays. Ce qui était vrai à l'époque et l'a été pendant plusieurs années, l'est-il encore aujourd'hui ? Depuis 1975, l'agriculture britannique a beaucoup évolué mais aussi la PAC elle-même. Une évaluation ${ }^{6}$ des transferts économiques et financiers

6. Elle est basée en partie sur le travail de Naudet $\mathrm{H}$. (2006). Les effets redistributifs de la politique agricole commune entre les États membres. Mémoire de fin d'études, Agrocampus de Rennes et ministère de l'Économie, des Finances et de l'Industrie. Diverses approches techniques sont possibles pour faire ces évaluations. Celle retenue ici dans le tableau 1 calcule d'abord le transfert budgétaire (aides à la production versées au pays moins sa contribution au financement de l'ensemble des aides via l'application de la clé budgétaire). Le surcoût net des importations est évalué directement à l'aide des restitutions unitaires (effet termes de l'échange) et couvre 18 principales lignes tarifaires. Une évaluation séparée a été faite pour les fonds au titre du développement rural. $C f$. Mahé P.-L., Naudet H., Roussillon-Montfort M.-A. (2006). Trading the Revision of the British Compensation for the Further CAP Reform: fair deal or a bad reason for a good purpose? Contribution présentée, au Workshop du Parlement européen on the "Budget Reform", Rome, novembre 2005. (opposé du coût de renationalisation) dus à la PAC pour la période 2001-2005 est résumée dans le tableau 1. La surcompensation éventuelle doit d'abord s'apprécier par l'écart entre le rabais et le coût de la PAC pour le Royaume-Uni ${ }^{7}$. Le rabais est en effet défini sur la base des recettes et des contributions financières, dont le solde net au titre du premier pilier de la PAC figure dans la première ligne. La surcompensation apparaît évidente et d'ampleur significative.

Les raisons de cette surcompensation sont nombreuses et viennent en partie de la régression de la part de la PAC dans les dépenses totales de l'Union européenne et du développement des politiques de cohésion qui ont peu de chance de bénéficier au RU dont le revenu par tête est passé de $91 \%$ de la moyenne communautaire en 1984 à $111 \%$ en 2003.

7. Même en prenant en compte l'effet termes de l'échange et les dépenses du développement rural (les plus conformes à l'économie publique), la surcompensation domine en moyenne (Mahé et al, op. cit.). 
Tableau 1. Surcompensation du coût de la PAC pour le Royaume-Uni (rabais moins le coût budgétaire de la PAC pour le RU, en millions $€$ )

\begin{tabular}{l|c|c|c|c|c}
\hline Années & 2001 & 2002 & 2003 & 2004 & 2005 \\
\hline (1) Transfert budgétaire net (1 ${ }^{\text {er }}$ pilier) & -2787 & -4066 & -3409 & -2984 & -3436 \\
\hline (2) Rabais & +7342 & +4933 & +5184 & +5272 & +5186 \\
\hline Surcompensation (2) - (1) & +4555 & +867 & +1775 & +2288 & +1750 \\
\hline
\end{tabular}

Source : voir note 5 .

En outre, le rabais a été protégé des conséquences des changements du mécanisme des ressources propres et des effets de l'élargissement, en excluant le Royaume-Uni d'une partie des dépenses liées à cet élargissement ${ }^{8}$. Ces dispositions d'un particularisme évident éloignent encore la nature du problème de la compensation de celui de la PAC elle-même, à moins de considérer que la défense énergique de la PAC traditionnelle par la France et de son extension aux nouveaux États membres est au cœur d'un conflit de visions du rôle de l'Europe en matière agricole. Là réside sans doute le nœud de l'affaire.

\section{De Charybde en Scylla ? Fin de la solidarité financière ou renationalisation?}

Les États membres de l'Union européenne ont toujours cherché un équilibre entre les bénéfices et les coûts de l'appartenance. La construction européenne a toujours subi les aléas de ce compromis. Un problème sérieux émerge quand les politiques qui en résultent sont inefficaces. Dans le cas d'une politique sectorielle spécifique comme la PAC, les moyens d'intervention touchent directement des intérêts catégoriels de certains groupes bien identifiés. Ces intérêts sont portés par les ministres et se mutent en intérêts nationaux dans les réunions du Conseil. Chaque ministre défend les programmes et les

8. Voir European Commission, 2004, p. 16 (Cf. note 4). dépenses qui bénéficient à ses mandants. Le coût financier étant externalisé sur les partenaires à cause de la solidarité financière, il n'y a pas ou peu d'incitation à comparer le bénéfice public au coût public de ces politiques. Un régulateur manque dans le processus de décision politique et l'on assiste à une surenchère de demandes d'intervention qui finissent par faire un paquet de compromis où l'intérêt général est mal représenté. Ce mécanisme institutionnel constitue un jeu appelé dilemme du prisonnier qui empêche l'émergence de la coopération et de décisions politiques favorables à l'intérêt public européen' .

L'importance prise par l'examen des soldes nets dans les décisions budgétaires et dans les réformes de la PAC illustre ce problème. La «fatigue politique » du plus grand contributeur net qu'est l'Allemagne se fait sentir de façon prégnante. Le RU, après avoir obtenu une compensation financière, a toujours freiné les réformes qui allaient toucher particulièrement ses agriculteurs, tout en remettant en cause la PAC elle-même. Le blocage en 1991 du premier projet de réforme de la PAC, qui introduisait la modulation sous le nom de dégressivité, est un exemple.

La poursuite d'un retour budgétaire de la PAC est aussi une constante de la position française. Elle est d'autant plus étonnante que l'élargissement et l'accroissement progressif des soutiens accordés aux

9. Voir par exemple Mahé P.-L., Roe T.-L. (1996). The Political Economy of 1992 CAP Reform. American Journal of Agricultural Economics, 78, décembre, p. 1314-1323. 
secteurs agricoles des nouveaux États membres ont finalement érodé l'avantage que la France retirait de la PAC (le solde devient négatif). Cette position n'est pas plus défendable au plan des principes que le lien fait par T. Blair entre les deux révisions (rabais et PAC). Elle a un coût politique qui obère la crédibilité de la France en Europe.

Cette volonté de la France de maintenir, à quelques nuances près, la PAC actuelle est promise à des lendemains difficiles. Le débat récurrent sur les politiques qui doivent être communes et celles qui peuvent rester nationales ou régionales a repris de la vigueur avec la stratégie de Lisbonne et la perte de crédibilité de la répartition des aides agricoles sur des bases historiques (droits à paiement unique, DPU), pratiquée dans certains pays dont la France. La recherche d'efficacité des dépenses communautaires devient un enjeu reconnu dans les instances communautaires. La nécessité d'une «valeur ajoutée » des dépenses communautaires par rapport à celles des États gagne en consensus. La seule poursuite d'intérêts nationaux particuliers perdra de la légitimité.

La définition du bon niveau de compétence et de financement des politiques peut puiser dans les apports des recherches sur le fédéralisme fiscal et les choix publics. Les questions à prendre en compte sont l'information, les mécanismes incitatifs vertueux, les modes de décision et le risque d'échec politique (équilibre politique biaisé par représentation d'intérêts particuliers), les économies d'échelles (par exemple recherche, infrastructures, défense...), les externalités globales (émissions $\mathrm{CO}_{2}$ ), l'étalement des risques et la redistribution aux fins de cohésion sociale. La direction générale à privilégier est de confier les biens publics locaux aux autorités locales et les biens publics européens aux instances supranationales, sauf en cas d'échec politique des décisions locales qui pourraient gagner à être encadrées par des règles décidées au niveau communautaire ${ }^{10}$. On retrouve le principe de subsidiarité qui reste toutefois ambigu concernant le financement.

S'agissant de l'agriculture, quels sont les Biens publics européens (BPE) ou les objectifs de cohésion relevant des politiques supranationales ? L'aspect social d'abord : le bilan de la PAC est peu convaincant car elle donne beaucoup aux grandes exploitations céréalières des régions les plus riches pour donner un peu aux petits agriculteurs des régions pauvres. Il est connu que poursuivre des objectifs de redistribution sociale à travers une politique sectorielle coûtera cher et manquera la cible des plus défavorisés. Les BPE liés à l'agriculture sont d'abord le marché unique et les avantages d'économies d'échelle qui en résultent, la sécurité alimentaire (en qualité et en quantité), le partage et l'étalement des risques naturels. La qualité environnementale à travers l'Europe comporte des aspects globaux et locaux. Les aménités paysagères ou la qualité de l'eau peuvent paraître locales, mais elles sont aussi une valeur d'option pour tous les Européens car ils peuvent un jour en profiter. Le maintien de la biodiversité a une valeur d'existence qui concerne à la fois l'ensemble des citoyens et ceux qui sont proches des milieux naturels concernés.

La question du bon niveau de compétence n'est pas simple, mais la PAC actuelle et son financement passent-ils bien le test ci-dessus ? Bien sûr, des corrections importantes ont été apportées mais l'instrument essentiel et le plus dispendieux en vigueur, le DPU, n'est pas cohérent avec les principes fondamentaux de niveau

10. Un cas concert est celui de la gestion des nitrates en Bretagne. L'équilibre politique local entre les groupes d'intérêt, avec des interférences nationales importantes, a toléré un niveau élevé de pollution, peu conforme à l'intérêt général. L'existence de décisions communautaires sur la protection des ressources en eau (Directives) donne un cadre juridique favorable à une meilleure maîtrise des pollutions. 
approprié de compétence et de mode de financement. Les clauses d'éco-conditionnalité et leur mise en œuvre ne suffissent pas à faire du DPU un instrument d'incitation à la fourniture des biens publics ruraux essentiels que sont la préservation de la nature et le maintien d'un espace rural ouvert et du patrimoine des paysages agraires. Les aides doivent être proportionnées à la fourniture de ces services environnementaux et territoriaux, et découplées de bases historiques dont la légitimité est mise à mal, entre autres par leur répartition très inégale et par la flambée actuelle des prix des produits de base.

Il en va différemment des aides du second pilier qui sont bien plus en cohérence avec les objectifs assignés aux politiques communautaires. Et pourtant ce sont ces politiques dites de développement rural, pour lesquelles un cofinancement national ou régional est requis. Les arguments économiques et politiques militeraient pour que ce soit l'inverse.

C'est le premier pilier qui devrait être cofinancé par les États membres, ce qui aurait pour grand avantage d'inciter les États bénéficiaires de la PAC à plus de responsabilité car leur retour budgétaire en serait atténué. Nous avons ainsi simulé une application d'un taux de cofinancement de $50 \%$ du premier pilier et constaté qu'il atténuerait fortement les écarts entre gagnants et perdants au jeu de la PAC et éviterait les effets pervers du mode actuel de financement du rabais britannique ${ }^{11}$.

Là encore les positions françaises répétées de protection de l'enveloppe financière du premier pilier sont difficilement soutenables à long terme.

$$
\begin{gathered}
* \\
* *
\end{gathered}
$$

Faute d'avoir une vision de la PAC basée sur des arguments scientifiques qui font consensus au niveau européen, la France ne pourra pas, quels que soient ses talents diplomatiques, faire prévaloir à long terme un point de vue basé sur une position d'intérêt national. À défaut d'une remise en cause de la solidarité financière dans le premier pilier et d'une remise à plat des aides, des pans entiers de la PAC finiront par disparaître ou être renationalisés sous la pression du consensus de bon usage des fonds publics européens. Là réside tout l'enjeu des discussions en cours sur le Bilan de santé et de celles à venir sur l'évolution et le financement de la PAC après 2013.

11. Voir le graphique 4 dans Mahé, Naudet, Roussillon-Montfort (2006). 